\title{
Optical coatings for gravitational wave detection
}

Gregory M. Harry, Helena Armandula, Eric Black, David R.M. Crooks, Gianpietro Cagnoli, et al.

Gregory M. Harry, Helena Armandula, Eric Black, David R.M. Crooks, Gianpietro Cagnoli, Martin M. Fejer, Jim Hough, Steven D. Penn, Sheila Rowan, Roger Route, Peter Sneddon, "Optical coatings for gravitational wave detection," Proc. SPIE 5527, Advances in Thin Film Coatings for Optical Applications, (29 September 2004); doi: 10.1117/12.555780

Event: Optical Science and Technology, the SPIE 49th Annual Meeting, 2004, Denver, Colorado, United States 


\title{
Optical coatings for gravitational-wave detection
}

\author{
Gregory M Harry ${ }^{a}$, Helena Armandula ${ }^{b}$, Eric Black ${ }^{b}$, D R M Crooks ${ }^{c}$, Gianpietro Cagnoli ${ }^{c}$, \\ Martin M Fejer ${ }^{d}$, Jim Hough ${ }^{c}$, Steven D Penn ${ }^{e}$, Sheila Rowan ${ }^{c}$, Roger Route ${ }^{d}$, Peter Sneddon ${ }^{c}$ \\ ${ }^{a}$ LIGO Laboratory, Massachusetts Institute of Technology, NW17-161, Cambridge MA, 02319, \\ USA; \\ ${ }^{b}$ LIGO Laboratory, California Institute of Technology, Pasadena CA, 91125, USA; \\ ${ }^{c}$ Department of Physics and Astronomy, The University of Glasgow, Glasgow G12 8QQ, \\ United Kingdom ; \\ ${ }^{d}$ Edward L Ginzton Laboratory, Stanford University, Stanford CA 94305, USA; \\ ePhysics Department, Hobart and William Smith Colleges, Geneva, NY 14456;
}

\begin{abstract}
Gravitational waves are a prediction of Einstein's General Theory of Relativity. Astrophysical events like supernova and binary neutron star inspirals are predicted to create potentially detectable waves. The Laser Interferometer Gravitational-wave Observatory (LIGO) is an experiment to detect these waves using Michelson interferometers with $4 \mathrm{~km}$ long arms. The effect of gravitational waves, even on an interferometer with such a long baseline, is extremely small, with mirror displacements around $10^{-18} \mathrm{~m}$. Reducing noise is thus a primary design criterion. For the next generation interferometers now being designed, thermal noise from the optical coatings of the interferometer mirrors could prove a problematic limiting noise source. Reducing the mechanical loss of these coatings to improve thermal noise, while preserving the sub-ppm optical absorption, low scatter, and high reflectivity needed in the interferometer is an important area of research.
\end{abstract}

Keywords: optical coatings, astronomy, interferometry

\section{INTRODUCTION}

In 1916, Albert Einstein published his General Theory of Relativity which improved upon Isaac Newton's description of gravity. This theory allows for a dynamic interaction between masses and the space-time in which they rest. The low-field, linear limit of General Relativity predicts waves which propagate through space-time analogously to electromagnetic waves predicted by Maxwell's equations. The gravitational wave is manifested as a strain, so the light travel time between two freely falling masses will change as the wave passes. The amplitude scale of the strain is set by the ratio

$$
G m v^{2} /\left(r c^{4}\right) \approx 10^{-21}\left(m / M_{\odot}\right)(v / c)^{2}(100 \mathrm{Mpc} / r)
$$

where $G$ is Newton's gravitational constant, $m$ is the source mass, $v$ is the velocity of the mass, $r$ is the distance to the source, $c$ is the speed of light, $M_{\odot}$ is the mass of the sun, and Mpc is a million parsecs. To get an appreciable amplitude, astronomical-sized masses moving at near the speed of light are needed. Even with that, a detectable wave at Earth is expected to have a strain of about $10^{-21}$, or about the width of a hair over the distance between the sun and the nearest star.

One method of detecting gravitational waves is to use a Michelson interferometer with two perpendicular arms, as shown in Fig. 1. Perpendicular arms allow for detection of the tensor field of the gravitational wave (one difference from the vector electromagnetic wave). The mirrors of the interferometer are hung as pendulums, to best approximate freely falling bodies in the interferometer's sensitive direction. To increase the laser power on the mirrors, each arm of the Michelson interferometer is a Fabry-Perot cavity while the entire path of the laser is in vacuum to minimize scatter. The signal comes from the displacement of the mirrors, so to get measurable displacement from the gravitational wave's strain, $4 \mathrm{~km}$ long arms are used. Even with this, the expected length change is only about $10^{-18} \mathrm{~m}$, so noise is the crucial design criterion.

Send correspondence to Gregory Harry, gharry@ligo.mit.edu 


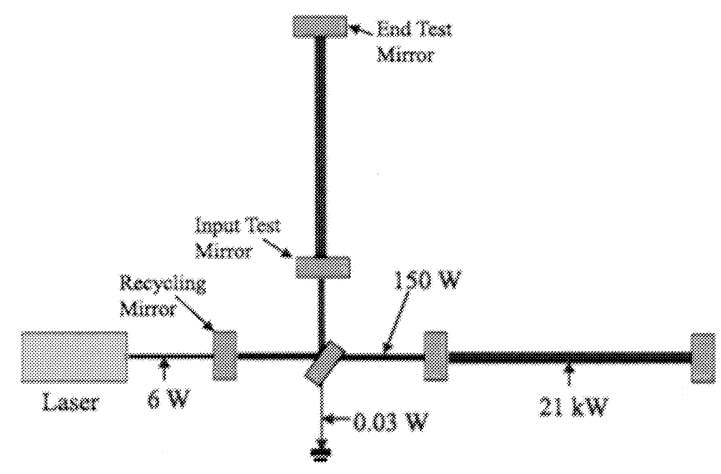

Figure 1. Schematic drawing of a LIGO interferometer. Light enters the system from the laser on the left, passes through the power recycling mirror, and is sent to both arms at the beamsplitter. Each arm cavity is formed from an input test mirror and an end test mirror. The laser power in each region is shown.

LIGO operates two observatories in the United States, one in Livingston, Louisiana (near Baton Rouge) and the second in eastern Washington state on the US Department of Energy Hanford reservation. Photos of the sites are shown in Fig. 2. There are a total of three interferometers, as the vacuum system at Hanford houses two with the second having half the arm length, $2 \mathrm{~km}$ rather than $4 \mathrm{~km}$. LIGO is part of an international network of gravitational wave observatories, with detectors in Germany (GEO 600), Italy (Virgo), and Japan (TAMA 300). These first generation interferometric detectors have a sensitivity such that a detection of gravitational waves is possible, but may not be probable. Next generation detectors with lower noise, including an Advanced LIGO, are now being designed. Advanced LIGO may be able to detect astronomical gravitational waves at a rate as high as one per day. ${ }^{1}$

\section{THE LIGO GRAVITATIONAL WAVE DETECTORS}

A brief description of the LIGO interferometers will be given here. A more detailed description can be found in a recent paper. ${ }^{2}$ Each LIGO detector is a Michelson interferometer with $4 \mathrm{~km}$ (or $2 \mathrm{~km}$ ) long Fabry-Perot arm cavities with finesse about 100 . The interferometer is held locked to a dark fringe at the output port, thus most of the laser light exits back towards the laser. To further increase power in the interferometer, an additional mirror is added at this port to reflect the light back into the system. This mirror forms an additional cavity with the arms known as the power recycling cavity. ${ }^{3}$ The design of Advanced LIGO adds an additional mirror
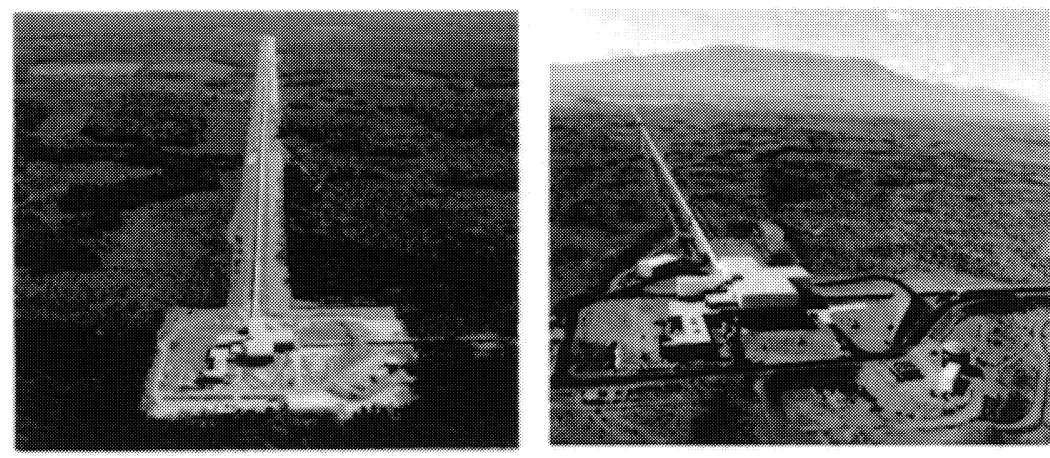

Figure 2. Photographs of the two LIGO sites; Livingston, Louisiana on the left and Hanford, Washington on the right. Each arm stretches $4 \mathrm{~km}$ from the corner station. 

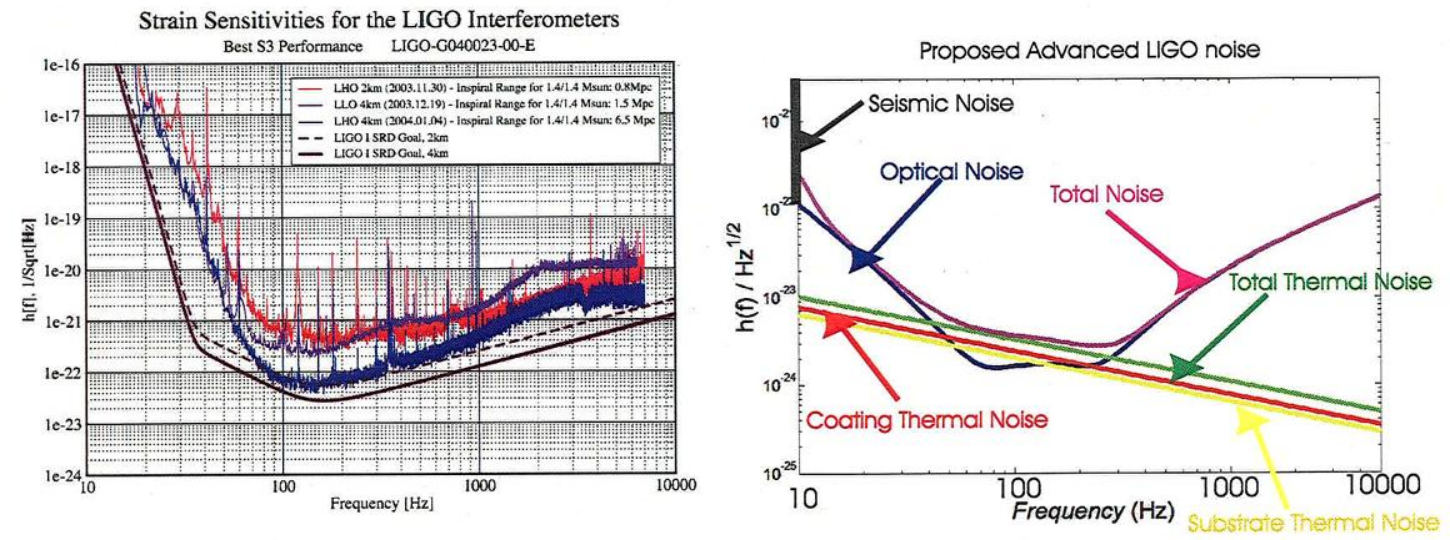

Figure 3. Design noise for the initial LIGO interferometers (on left). The solid line is the design noise for the $4 \mathrm{~km}$ long interferometers, while the dashed line is for the $2 \mathrm{~km}$ long one. Also shown is the measured noise in all three initial LIGO interferometers as of March 2004. On the right is the design sensitivity of the proposed Advanced LIGO interferometer. Note the difference in Y-axis offset between the two graphs; Advanced LIGO will have about a factor of 15 lower noise compared with initial LIGO in most bands.

at the output port, forming a further cavity. This allows the sensitivity of the interferometer to be tuned, so the most sensitive band can occur at different frequencies and/or with different bandwidths. This is known as signal recycling ${ }^{3}$ and is currently being used in GEO 600.

The design noise for initial LIGO is shown in Fig. 3, which also shows the measured noise in each LIGO interferometer as of March 2004. All the LIGO mirrors are isolated from ground motion by multi-stage vibration isolation stacks to reduce seismic noise. The pendulum suspensions and careful clamping and connection to the mirrors helps keep down the thermal noise. The laser is carefully controlled so frequency and amplitude noise are below the shot noise level.

Fig. 3 also shows the planned sensitivity of Advanced LIGO, which is being designed now. In Advanced LIGO, the mirrors are better isolated from seismic noise, so that it no longer dominates above about $10 \mathrm{~Hz}$. The addition of the signal recycling mirror mixes the shot noise and the radiation pressure into a single optical noise, which is dominant in most of the low and high frequency bandwidth. The Fabry-Perot arms also will have higher finesse, about 1000, for increased stored power. Between about $40 \mathrm{~Hz}$ and a few hundred Hertz, the most sensitive region, thermal noise from the mirrors sets the sensitivity limit. This can be divided into a contribution from the mirror substrate and the mirror coating. The graph in Fig. 3 assumes silica mirrors with a silica/tantala dielectric coating. Sapphire mirrors are also under consideration for use in Advanced LIGO. The frequency dependence of substrate thermal noise is steeper in sapphire, as it is dominated by thermoelastic loss. ${ }^{4}$ Coating thermal noise will still cause an increase in noise at the most sensitive bandwidth in a sapphire interferometer, but over a smaller band.

\section{COATING THERMAL NOISE}

Thermal noise is caused by mechanical dissipation in a system, according the the Fluctuation-Dissipation Theorem of Callen and Green. ${ }^{5}$ This theorem can be applied to the problem of thermal noise in a coated mirror being monitored by a Gaussian shaped laser beam ${ }^{6}$ to give

$$
S_{x}(f)=2 k_{B} T \phi_{\text {eff }}(1-\sigma) /\left(\pi^{3 / 2} f w Y\right)
$$

where $S_{x}(f)$ is the power spectral density of position noise, $k_{B}$ is Boltzmann's constant, $T$ is the temperature, $\sigma$ is the Poisson ratio of the substrate material, $w$ is the half-width of the Gaussian laser beam, and $\phi_{\text {eff }}$ is the 
effective loss angle, given by

$$
\begin{aligned}
\phi_{\mathrm{eff}}= & \phi+d /\left(\sqrt{\pi} w Y_{\perp}\right) \\
& \left(\left(Y /\left(1-\sigma_{\perp}\right)-2 \sigma_{\perp}^{2} Y Y_{\|} /\left(Y_{\perp}\left(1-\sigma^{2}\right)\left(1-\sigma_{\|}\right)\right)\right) \phi_{\perp}\right. \\
& +Y_{\|} \sigma_{\perp}(1-2 \sigma) /\left(\left(1-\sigma_{\|}\right)(1-\sigma)\right)\left(\phi_{\|}-\phi_{\perp}\right) \\
& \left.+Y_{\|} Y_{\perp}(1+\sigma)(1-2 \sigma)^{2} /\left(Y\left(1-\sigma_{\|}^{2}\right)(1-\sigma)\right) \phi_{\|}\right),
\end{aligned}
$$

where $d$ is the thickness of the coating, $Y, \sigma$ and $\phi$ are the Young's moduli, Poisson's ratios and loss angles of the substrate (no subscript), the coating for stresses perpendicular $(\perp)$ and parallel $(\|)$ to the optic face. For simplicity, if all the Poisson ratios are small, Eq. 3 can be written

$$
\phi_{\mathrm{eff}}=\phi_{\mathrm{sub}}+d /(\sqrt{\pi} w)\left(Y / Y_{\perp} \phi_{\perp}+Y_{\|} / Y \phi_{\|}\right)
$$

The values for these elastic constants can be calculated from the parameters of the isotropic materials used to make the multilayer coating $\left(\mathrm{SiO}_{2}\right.$ and $\mathrm{Ta}_{2} \mathrm{O}_{5}$ for the initial LIGO coatings). With two alternating materials, these values are

$$
\begin{aligned}
Y_{\perp} & =\left(d_{1}+d_{2}\right) /\left(d_{1} / Y_{1}+d_{2} / Y_{2}\right) \\
Y_{\|} & =\left(Y_{1} d_{1}+Y_{2} d_{2}\right) /\left(d_{1}+d_{2}\right) \\
\sigma_{\perp} & =\left(\sigma_{1} Y_{1} d_{1}+\sigma_{2} Y_{2} d_{2}\right) /\left(Y_{1} d_{1}+Y_{2} d_{2}\right) \\
\phi_{\perp} & =Y_{\perp}\left(\phi_{1} d_{1} / Y_{1}+\phi_{2} d_{2} / Y_{2}\right) \\
\phi_{\|} & =\left(Y_{1} \phi_{1} d_{1}+Y_{2} \phi_{2} d_{2}\right) /\left(Y_{\|}\left(d_{1}+d_{2}\right)\right)
\end{aligned}
$$

where subscripts 1 and 2 refer to the two different materials. For $\sigma_{\|}$the equivalent equation is quite complicated. Simply averaging the two material Poisson ratios agrees with a numerical solution to better than $5 \%$ for the case of a $\mathrm{SiO}_{2} / \mathrm{Ta}_{2} \mathrm{O}_{5}$ coating with thicknesses appropriate for high reflectivity at $1.064 \mu \mathrm{m}$, the wavelength of the initial LIGO laser.

This analysis assumes the loss in the coating and substrate to be due to internal friction in the materials. Thermoelastic loss ${ }^{7}$ can also occur, where contraction and expansion of the material causes heat flow between the coating and the substrate ${ }^{8,9}$ or different parts of the substrate. ${ }^{4}$ To minimize thermoelastic loss between the coating and the substrate, the thermal expansion coefficient and Young's modulus should be matched across the interface. Eq. 4 shows that, for roughly equal $\phi_{\perp}$ and $\phi_{\|}$, matching Young's moduli is also desirable for reducing the Brownian thermal noise in Eq. 2. Here we assume this matching has been done well enough that the coating thermal noise is dominated by Brownian noise and coating thermoelastic noise is the smaller of the two. This will add a constraint on the thermal expansion coefficient of the coating, and may require different coatings with different thermal expansions for silica and sapphire substrates.

\section{COATING RESEARCH FOR ADVANCED LIGO}

To be acceptable for Advanced LIGO, the coating must satisfy stringent optical as well as mechanical/thermal properties. The mirrors must define high finesse Fabry-Perot arms in the interferometer as well as have low thermal noise. Table 1 shows a list of coating requirements for Advanced LIGO as well as the currently demonstrated values, either in initial LIGO or in laboratory research samples. The thermal expansion coefficients of the coating and the substrate (either silica or sapphire) would ideally be matched to eliminate coating thermoelastic noise. Matching of the coating and substrate Young's moduli helps to minimize Brownian thermal noise, but lower coating loss angle is the primary concern. Greater optical loss (absorption, scatter, and transmission) than in Table 1 can possibly be made up for by higher laser power. Research towards an Advanced LIGO coating has focussed on reducing the loss angle, partially because the optical requirements have this work-around, the substrate material has not been chosen, and because so little is known about mechanical loss in dielectric coatings. 
Table 1. Coating requirements for Advanced LIGO. These are the necessary values to reach the high finesse and low thermal noise needed. The demonstrated values have not all been shown on a single coating. In addition, the thermal expansion coefficient and Young's modulus of the coating must be matched to that of the substrate within a factor of about 3 .

\begin{tabular}{|l|r|r|}
\hline Parameter & adv. LIGO requirement & Demonstrated Value \\
\hline Loss Angle $\phi_{\|}$ & $5 \times 10^{-5}$ & $1.5 \times 10^{-4}$ \\
\hline Optical Absorption & $0.5 \mathrm{ppm}$ & $1 \mathrm{ppm}$ \\
\hline Scatter & $2 \mathrm{ppm}$ & $20 \mathrm{ppm}$ \\
\hline Thickness Uniformity & $10^{-3}$ & $8 \times 10^{-3}$ \\
\hline Transmission & $5 \mathrm{ppm}$ & $5.5 \mathrm{ppm}$ \\
\hline Transmission Matching & $5 \times 10^{-3}$ & $1 \times 10^{-2}$ \\
\hline
\end{tabular}

The mechanical loss in test coatings is determined by measuring the quality factor, $Q$, of normal modes of silica disks both before and after coating. Two disk geometries have been used, both $7.62 \mathrm{~cm}$ in diameter, with one $2.54 \mathrm{~cm}$ and the other $0.254 \mathrm{~cm}$ thick. The lowest mode of the thin disks is at $2.7 \mathrm{kHz}$ and of the thick disk is at about $21 \mathrm{kHz}$. The thick disks are suspended by a wire loop from a rigid platform and the thin disks are welded to a silica fiber below a silica mass, all in vacuum. This is to minimize mechanical loss from any effect, such as rubbing friction, other than internal friction in the samples. Normal modes of the silica samples are excited using a comb capacitor, and the ringdowns of modes are monitored using either a simple Michelson interferometer or a birefringence readout. A sketch of the experimental setups for both samples is shown in Fig. 4 and details of this technique are discussed in recent papers. ${ }^{10,11}$

The loss angle of the coating, $\phi_{\|}$, is determined from the modal $Q$ from

$$
1 / Q_{\text {coated }}=1 / Q_{\text {uncoated }}+\phi_{\|} E_{\text {coating }} / E_{\text {total }}
$$

where $Q_{\text {coated }}$ is the measured modal $Q$ of the coated sample, $Q_{\text {uncoated }}$ is the measured modal $Q$ of the uncoated sample, $E_{\text {coating }}$ is the total elastic energy stored in the coating for the particular mode, $E_{\text {total }}$ is the total elastic energy in the mode, and it is only $\phi_{\|}$of the coating that enters because the free boundary condition on the sample surface ensures there is no stress perpendicular to the face.

The first round of $Q$ measurements was to determine the cause of the loss in $\mathrm{SiO}_{2} / \mathrm{Ta}_{2} \mathrm{O}_{5}$ coatings. Possibilities explored were interaction between the coating and the substrate, interaction between the different layers within

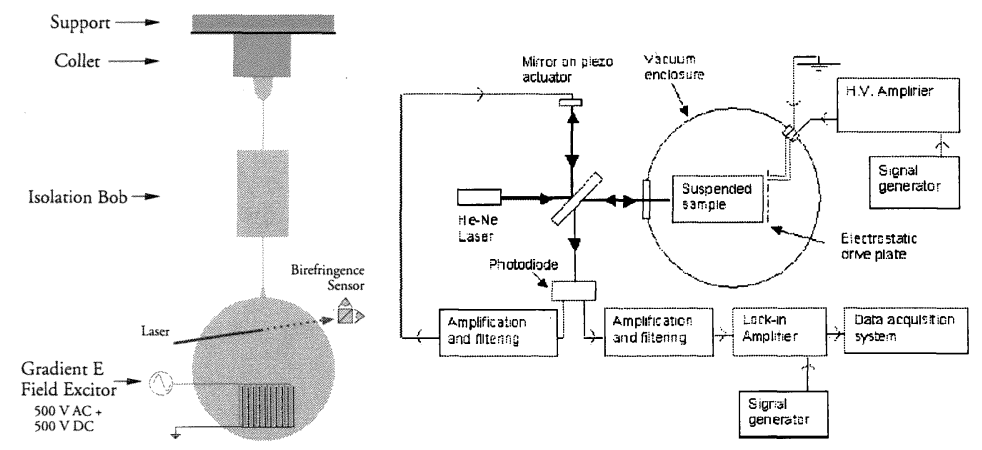

Figure 4. On the left, a schematic of the thin disk experimental setup, showing the welded suspension and the birefringence readout. On the right, a schematic of the thick disk experimental setup, showing the interferometer readout and related electronics. 
Table 2. Results of first round of mechanical loss tests. The goal was to determine the source of loss in $\mathrm{SiO}_{2} / \mathrm{Ta}_{2} \mathrm{O}_{5}$ coatings.

\begin{tabular}{|r|r|r|r|}
\hline Total Layers & $\mathrm{SiO}_{2}$ Optical Thickness & $\mathrm{Ta}_{2} \mathrm{O}_{5}$ Optical Thickness & Loss angle $\phi_{\|}$ \\
\hline 2 & $\lambda / 4$ & $\lambda / 4$ & $2.7 \pm 0.7 \times 10^{-4}$ \\
\hline 30 & $\lambda / 4$ & $\lambda / 4$ & $2.6 \pm 0.7 \times 10^{-4}$ \\
\hline 60 & $\lambda / 8$ & $\lambda / 8$ & $2.7 \pm 0.5 \times 10^{-4}$ \\
\hline 30 & $\lambda / 8$ & $3 \lambda / 8$ & $3.7 \pm 0.5 \times 10^{-4}$ \\
\hline 30 & $3 \lambda / 8$ & $\lambda / 8$ & $1.9 \pm 0.2 \times 10^{-4}$ \\
\hline
\end{tabular}

the coating, and internal friction in the $\mathrm{SiO}_{2}$ and/or $\mathrm{Ta}_{2} \mathrm{O}_{5}$. A series of samples were coated by SMA/Virgo (now LMA/Virgo) in Lyon, France. The first samples had only a two layer coating, one $\mathrm{SiO}_{2}$ and one $\mathrm{Ta}_{2} \mathrm{O}_{5}$, to test for coating-substrate interactions. The second samples had a baseline 30 layer coating applied. The third samples had 60 layers, but each layer only half as thick as the baseline, to test for coating layer interface effects. The fourth samples had additional $\mathrm{Ta}_{2} \mathrm{O}_{5}$ and the fifth had additional $\mathrm{SiO}_{2}$, to test for internal friction effects from the individual materials. The results of these tests are shown in Table 2. This experiment is more fully described in a recent paper. ${ }^{12}$

The results of these tests clearly support the conclusion that it is internal friction from the $\mathrm{SiO}_{2}$ and $\mathrm{Ta}_{2} \mathrm{O}_{5}$ that determines the loss in the coating and that $\mathrm{Ta}_{2} \mathrm{O}_{5}$ is the chief contributor. Using the results from the last two set of samples with the differing amounts of $\mathrm{SiO}_{2}$ and $\mathrm{Ta}_{2} \mathrm{O}_{5}$, individual loss angles can be determined for these materials (assuming a structural, i.e. frequency independent, model of internal friction);

$$
\begin{aligned}
\phi_{\mathrm{SiO} 2} & =0.5 \pm 0.3 \times 10^{-4} \\
\phi_{\mathrm{Ta} 2 \mathrm{O} 5} & =4.4 \pm 0.2 \times 10^{-4} .
\end{aligned}
$$

We also measured 30 layer, $\lambda / 4 \mathrm{SiO}_{2} \lambda / 4 \mathrm{Ta}_{2} \mathrm{O}_{5}$ coatings coated by MLD Technologies of Mountain View, CA, USA and got similar results. Further measurements have been made on $\mathrm{Al}_{2} \mathrm{O}_{3} / \mathrm{Ta}_{2} \mathrm{O}_{5}$ and $\mathrm{SiO}_{2} / \mathrm{Nb}_{2} \mathrm{O}_{5}$ coatings. Preliminary analysis of the $\mathrm{Al}_{2} \mathrm{O}_{3} / \mathrm{Ta}_{2} \mathrm{O}_{5}$ coatings is consistent with the findings that the loss from the $\mathrm{Ta}_{2} \mathrm{O}_{5}$ component is greater than that of the $\mathrm{Al}_{2} \mathrm{O}_{3}$. Work is in progress to extract the individual material loss angles for $\mathrm{Al}_{2} \mathrm{O}_{3}$ and $\mathrm{Nb}_{2} \mathrm{O}_{5}$ from this data. If sapphire mirrors are chosen for Advanced LIGO, $\mathrm{Al}_{2} \mathrm{O}_{3}$ may be the preferred low index material for better matching of elastic and thermal properties.

By looking at the coating loss in the $\mathrm{SiO}_{2} / \mathrm{Ta}_{2} \mathrm{O}_{5}$ coatings mode by mode, it is possible to explore the frequency dependence of the loss. This results in ${ }^{13}$

$$
\begin{aligned}
\phi_{\mathrm{SiO} 2} & =(0.4 \pm 0.3) \times 10^{-4}+f(2.7 \pm 0.9) \times 10^{-9} \\
\phi_{\mathrm{Ta} 2 \mathrm{O} 5} & =(4.2 \pm 0.4) \times 10^{-4}+f(0.4 \pm 0.9) \times 10^{-9}
\end{aligned}
$$

These results depend strongly on just a few modal $Q$ 's, however. Work is in progress to measure higher frequency modes on the thin samples, to span the frequency gap between the lowest modes of the thin and thick samples.

Thermal noise from a $\mathrm{SiO}_{2} / \mathrm{Ta}_{2} \mathrm{O}_{5}$ coating has been directly measured, both in Japan ${ }^{14}$ and by LIGO's Thermal Noise Interferometer (TNI) ${ }^{15}$ The TNI is a Michelson interferometer with Fabry-Perot arms similar to LIGO, but with an arm length of $1 \mathrm{~cm}$ rather than $4 \mathrm{~km}$. This requires a much smaller spot size, $0.15 \mathrm{~mm}$ in the TNI compared to $40 \mathrm{~mm}$ in initial LIGO and a planned $60 \mathrm{~mm}$ in Advanced LIGO. The mirrors of the TNI are silica substrates with $4.26 \mu \mathrm{m}$ of a $\mathrm{SiO}_{2} / \mathrm{Ta}_{2} \mathrm{O}_{5}$ coating done by Research Electro Optics of Boulder, CO, USA. Noise data from the TNI is shown in Fig. 5. Between $500 \mathrm{~Hz}$ and $6 \mathrm{kHz}$, the TNI noise has the correct frequency dependence of $1 / \sqrt{f}$ and is consistent with loss angles for the coating of $\phi_{\perp}=1.2 \times 10^{-4}$ and $\phi_{\|}=4.2 \times 10^{-4}$, or $\phi_{\mathrm{Ta} 2 \mathrm{O} 5}=5.1 \times 10^{-4}$ assuming $\phi_{\mathrm{SiO} 2}=5 \times 10^{-5}$.

There are now additional experiments going on to try to reduce the loss in the coating, especially focussed on the high index material. Results in progress for this work are shown in Table 3. Working with LMA/Virgo, 


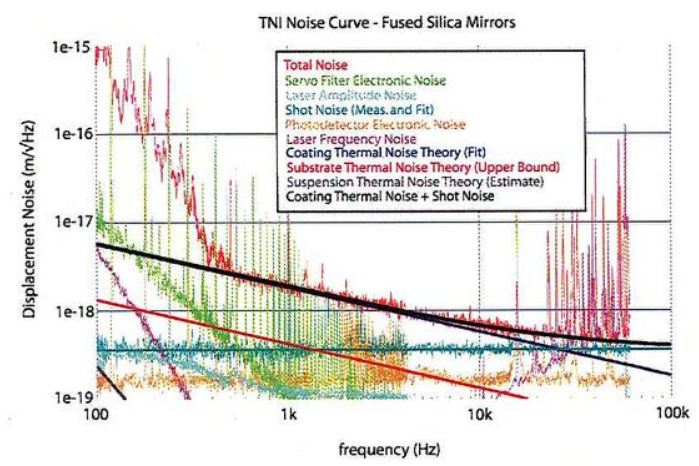

Figure 5. Direct measurement of coating thermal noise in LIGO's Thermal Noise Interferometer (TNI). These direct measurements allow a check on the inferred coating loss angles from $\mathrm{Q}$ measurements. The noise between about $500 \mathrm{~Hz}$ and $6 \mathrm{kHz}$ is due to thermal noise from the coating.

Table 3. Results of mechanical loss tests on $\mathrm{SiO}_{2} / \mathrm{TiO}_{2}$-doped $\mathrm{Ta}_{2} \mathrm{O}_{5}$ coatings. All coatings were 30 layers, with $\lambda / 4$ optical thickness in each layer.

\begin{tabular}{|c|r|}
\hline Concentration of $\mathrm{TiO}_{2}$ & Loss angle $\phi_{\|}$ \\
\hline None & $2.7 \pm 0.5 \times 10^{-4}$ \\
\hline Low & $1.8 \pm 0.2 \times 10^{-4}$ \\
\hline High & $1.6 \pm 0.2 \times 10^{-4}$ \\
\hline
\end{tabular}

$\mathrm{TiO}_{2}$ has been added as a dopant to $\mathrm{Ta}_{2} \mathrm{O}_{5}$. Increasing concentrations of $\mathrm{TiO}_{2}$ reduce the mechanical loss in $\mathrm{Ta}_{2} \mathrm{O}_{5}$. There are plans to pursue even higher concentration of $\mathrm{TiO}_{2}$. These $\mathrm{TiO}_{2}$-doped $\mathrm{Ta}_{2} \mathrm{O}_{5}$ coatings have had only about $1 \mathrm{ppm}$ of optical absorption at the two concentrations developed. Effort has gone into replacing the $\mathrm{Ta}_{2} \mathrm{O}_{5}$ with $\mathrm{HfO}_{2}$, although no satisfactory coatings have been made to date. Plans are being developed with CSIRO Telecommunications and Industrial Physics in Sydney, Australia to try to improve the stoichiometry of the $\mathrm{Ta}_{2} \mathrm{O}_{5}$ and to determine its effect on mechanical loss. There are also plans to pursue different annealing cycles of the coated samples.

There are other suggestions for reducing coating thermal noise in Advanced LIGO, beyond finding a lower mechanical loss coating. One is to use corner reflectors, rather than face-coated optics, ${ }^{16}$ taking advantage of total internal reflection. The other is to have an additional short cavity where the end test masses are now, with each mirror having a thinner coating than the traditional design. ${ }^{17}$ Both these ideas would represent fairly large changes to the proposed design, and further research is necessary before they could be implemented.

\section{CONCLUSION}

Directly measuring gravitational waves is one of the great challenges of experimental physics for the $21^{\text {st }}$ century. The required sensitivity is so great, that much effort must be put into noise reduction. For the next generation interferometric detectors, coating thermal noise will define the noise floor and thereby the astronomical reach of the instrument. Developing optical coating with low mechanical loss, low optical loss, and high reflectivity has become one of the central goals in experimental gravitational physics.

Many techniques to reduce mechanical loss in suitable coatings have been pursued, but much more work remains to be done. Input from material scientists, optical engineers, coating technologists, and others with specialized knowledge is crucial for proper guidance of this research. The LIGO Scientific Collaboration is very interested in forming collaborations with researchers who have knowledge, interest, and experience with optical coatings. 


\section{ACKNOWLEDGMENTS}

The LIGO Observatories were constructed by the California Institute of Technology and Massachusetts Institute of Technology with funding from the National Science Foundation under cooperative agreement PHY 9210038. The LIGO Laboratory operates under cooperative agreement PHY-0107417. This paper has been assigned LIGO Document Number LIGO-P040023-00-D.

\section{REFERENCES}

1. K. S. Thorne and C. Cutler, "An overview of gravitational wave sources," in Proceedings of 16th International Conference on General Relativity and Gravitation, N. T. Bishop and S. D. Maharaj, eds., pp. 72-112, World Scientific, Singapore, 2002.

2. B. Abbott and et al., "Detector description and performance for the first coincidence observations between LIGO and GEO," Nuclear Inst. and Methods in Physics Research A 517/1-3, pp. 154-179, 2004.

3. B. J. Meers, "Recycling in a laser-interferometric gravitational-wave detector," Phys. Rev. D 38, p. 2317, 1988.

4. V. B. Braginsky, M. L. Gorodetsky, and S. P. Vyatchanin, "Thermodynamical fluctuations and photothermal shot noise in gravitational wave antennae," Phys. Lett. A 264, pp. 1-10, 1999.

5. H. B. Callen and R. F. Greene, "On a theorem of irreversible thermodynamics," Phys. Rev. 86, pp. 702-710, 1952.

6. Y. Levin, "Internal thermal noise in the LIGO test masses: A direct approach," Phys. Rev. D 57, pp. 659663, 1998.

7. C. Zener, "Internal friction in solids. I. Theory of internal friction in reeds," Phys. Rev. 52, p. 230, 1937.

8. M. M. Fejer, S. Rowan, G. Cagnoli, D. R. M. Crooks, A. Gretarsson, G. M. Harry, J. Hough, S. D. Penn, P. H. Sneddon, and S. P. Vyatchanin, "Thermoelastic dissipation in inhomogeneous media: loss measurements and displacement noise in coated test masses for interferometric gravitational wave detectors," Phys. Rev. $D, 2003$. In press.

9. V. B. Braginsky and S. P. Vyatchanin, "Thermodynamical fluctuations in optical mirror coatings," Phys. Lett. A 312/3-4, pp. 224-255, 2003.

10. G. M. Harry, A. M. Gretarsson, P. R. Saulson, S. E. Kittelberger, S. D. Penn, W. J. Startin, S. Rowan, M. M. Fejer, D. R. M. Crooks, G. Cagnoli, J. Hough, and N. Nakagawa, "Thermal noise in interferometric gravitational wave detectors due to dielectric optical coatings," Class. Quantum Grav. 19, pp. 897-917, 2002.

11. D. R. M. Crooks, P. Sneddon, G. Cagnoli, J. Hough, S. Rowan, M. M. Fejer, E. Gustafson, R. Route, N. Nakagawa, D. Coyne, G. M. Harry, and A. M. Gretarsson, "Excess mechanical loss associated with dielectric mirror coatings on test masses in interferometric gravitational wave detectors," Class. Quantum Grav. 19(5), pp. 883-896, 2002.

12. S. D. Penn, P. H. Sneddon, H. Armandula, J. C. Betzweiser, G. Cagnoli, J. Camp, D. R. M. Crooks, M. M. Fejer, A. M. Gretarsson, G. M. Harry, J. Hough, S. E. Kittelberger, M. J. Mortonson, R. Route, S. Rowan, and C. C. Vassiliou, "Mechanical loss in tantala/silica dielectric mirror coatings," Class. Quantum Grav. 20, pp. 2917-2928, 2003.

13. D. R. M. Crooks, G. Cagnoli, M. M. Fejer, A. M. Gretarsson, G. Harry, J. Hough, N. Nakagawa, S. Penn, R. Route, S. Rowan, and P. H. Sneddon, "Experimental measurements of coating mechanical loss factors," Class. Quantum Grav. 21, pp. S1059-S1065, 2004.

14. K. Numata, "Wide-band direct measurement of thermal fluctuations in an interferometer," Phys. Rev. Lett. 91(26), p. 260602, 2003.

15. E. D. Black, A. Villar, K. Barbary, A. Bushmaker, J. Heefner, S. Kawamura, F. Kawazoe, L. Matone, S. Meidt, S. R. Rao, K. Schulz, M. Zhang, and K. G. Libbrecht, "Direct observation of broadband coating thermal noise in a suspended interferometer," Phys. Lett. A, 2004. Accepted for publication 25 May 2004.

16. V. B. Braginsky and S. P. Vyatchanin, "Corner reflectors and quantum-non-demolition measurements in gravitational wave antennae," Phys. Lett. A 324/5-6, pp. 345-360, 2004.

17. F. Y. Khalili, "Reducing the mirrors coating noise in laser gravitational-wave antennae by means of double mirrors," Phys. Lett. A, 2004. 